\title{
Alcohol y tabaco en adolescentes españoles y mexicanos y su relación con la actividad físico-deportiva y la familia
}

\author{
Jorge Ruiz-Risueño Abad, ${ }^{1}$ Francisco Ruiz-Juan ${ }^{1}$ \\ y Jorge Isabel Zamarripa Rivera ${ }^{2}$
}

Forma de citar Ruiz-Risueño Abad J, Ruiz-Juan F, Zamarripa Rivera JI. Alcohol y tabaco en adolescentes españoles y mexicanos y su relación con la actividad físico-deportiva y la familia. Rev Panam Salud Publica. 2012; 31(3):211-20.

RESUMEN Objetivo. Identificar la influencia del consumo de alcohol o tabaco junto a variables independientes (sexo y edad; tiempo y actividades de ocio; motivaciones, patrones, modalidades y finalidades físico-deportivas; $y$ conductas del entorno familiar) en la actividad físico-deportiva de alumnos de educación secundaria obligatoria en España y México.

Métodos. 3249 alumnos españoles y 1083 alumnos mexicanos de entre 12 y 16 años respondieron un cuestionario sobre hábitos físico-deportivos, consumo de bebidas alcohólicas o tabaco e influencia del entorno familiar. Se realizó un análisis descriptivo y una regresión logística multinomial para averiguar la capacidad predictiva de las variables incluidas en el modelo.

Resultados. En ambos países el consumo aumenta con la edad. Comparativamente existe mayor prevalencia entre los estudiantes españoles a la hora de consumir sustancias adictivas. Los estudiantes españoles físicamente activos tienen menos probabilidades de consumir alcohol y tabaco; entre los mexicanos no se encuentran diferencias significativas al respecto. Los resultados reflejan que en España las mujeres consumen bebidas alcohólicas y fuman con mayor frecuencia. Los varones españoles, sin embargo, lo hacen de manera esporádica pero en mayores cantidades. En México, los adolescentes consumen alcohol más regularmente que las adolescentes, pero no existen diferencias en la cantidad.

Conclusiones. En España, la práctica de actividades físico-deportivas se relaciona claramente con un menor consumo. Ser físicamente activo actúa como agente protector ante hábitos no saludables. En los adolescentes mexicanos no se ha encontrado una relación significativa entre estos factores. Tanto en España como en México, los hermanos y los padres son los familiares que mayor influencia ejercen respecto del consumo de alcohol y tabaco. Además, se ha comprobado la tendencia a un consumo simultáneo de ambas sustancias.

Palabras clave Consumo de bebidas alcohólicas; tabaquismo; adolescente; familia; deportes; España; México.

Según la OMS (1), el tabaquismo y el alcoholismo son las drogodependencias más extendidas en nuestra sociedad, y representan, respectivamente, la primera

\footnotetext{
1 Facultad de Ciencias del Deporte, Universidad de Murcia, Murcia, España. La correspondencia se debe enviar a Jorge Ruiz-Risueño Abad, jorgerichi@um.es

2 Facultad de Organización Deportiva, Universidad Autónoma de Nuevo León, Nuevo León, México.
}

y la tercera causa de años de vida con discapacidades. El Observatorio Español sobre Drogas asegura en su último informe que la mayoría de los españoles consume bebidas alcohólicas esporádica o habitualmente (2). En 2008, 72,9\% de estudiantes de entre 14 y 18 años ya había consumido bebidas alcohólicas y $14,8 \%$ se consideraba fumador habitual.
En México, el Observatorio Mexicano de Tabaco y Alcohol determinó que el porcentaje de jóvenes de entre 12 y 18 años consumidores tanto de alcohol como de tabaco llegaba a 30\% en 2003 (3).

La adolescencia es una etapa clave; en ella se conforma el estilo de vida. Durante su desarrollo aumenta la tendencia al consumo, pero si no se adop- 
tan hábitos nocivos durante esta etapa, es menos probable que se los adquiera una vez alcanzada la etapa adulta (4). La prevalencia de conductas saludables o perjudiciales depende en gran medida de las rutinas adoptadas en esta época de cambios individuales y sociales $(5,6)$. Es muy posible que un adolescente físicamente activo y con invariables hábitos saludables conserve esta línea positiva alcanzada su madurez, al igual que es muy viable que uno sedentario o con un estilo de vida poco saludable lo mantenga durante años $(7,8)$.

Los adolescentes componen el colectivo social más vulnerable, incomprendido y desprotegido. A pesar de que tanto las leyes españolas como las mexicanas prohíben la compra de alcohol y tabaco a menores de 18 años, se ha demostrado que el inicio en la adicción se produce antes de alcanzar esta edad (4-9). Es vital evitar una iniciación prematura, así como potenciar procesos de ayuda estableciendo políticas protectoras más enérgicas y eficaces (4-10).

La actividad física ha sido concebida casi siempre como una opción recomendable para reducir la probabilidad de consumir sustancias tóxicas: facilita lugares de encuentro, ayuda en las relaciones entre iguales, proporciona un entorno apropiado para establecer hábitos de vida saludable y es un medio para promocionar la salud (11-16); sin embargo, esta no es una opinión generalizada. También ha sido descrita por algunos autores como una actividad que favorece la adquisición de prácticas insanas (17).

Varios estudios asocian los deportes colectivos con el abuso de alcohol o tabaco (18-20). El grupo de iguales cobra especial relevancia entre adolescentes ya que es en esta época cuando se construye y se define la identidad personal. A la hora de buscar aceptación social, los adolescentes son especialmente sensibles a la influencia de los otros, a la interacción y a las presiones entre semejantes (21-23).

Se muestra con esto una relación con la teoría de la autodeterminación (2426). Por un lado, la motivación extrínseca alude a que la práctica de actividades físico-deportivas puede estar influenciada por motivos que no están ligados a la propia actividad (relaciones satisfactorias y aceptación social). Por otro lado, la motivación intrínseca, cuya práctica está influenciada por motivos propios a dicha actividad (sentir placer y satisfacción practicando o compitiendo).
La influencia familiar es la variable que con más insistencia se plantea en trabajos referidos a factores de riesgo, y juega un papel fundamental para explicar la aparición de diversas conductas (27). Los hábitos de familiares y personas cercanas al adolescente influyen para fijar, mantener o eliminar sus pautas de comportamiento (28-30). Las circunstancias externas y exigencias de su entorno son un claro condicionante.

Otro factor a tener en cuenta es el consumo conjunto de alcohol y tabaco. El riesgo de enfermedad y muerte es mucho mayor cuando se suman ambas adicciones (31). De hecho, los fumadores tienen un mayor grado de dependencia alcohólica. El alcoholismo es 10 veces más común en fumadores que en no fumadores $(9,32)$.

El objetivo del trabajo es conocer la prevalencia de consumo de alcohol y tabaco de dos muestras representativas de estudiantes de educación secundaria obligatoria (ESO) de España y México, y compararlas prestando especial atención a variables como sexo y edad; tiempo y actividades de ocio; motivaciones, patrones, modalidades y finalidades físico-deportivas; $\mathrm{y}$ conductas del entorno familiar.

Se pretende identificar aquellos factores de riesgo que pudieran facilitar o fomentar el consumo, así como generar estrategias de prevención.

\section{MATERIALES Y MÉTODOS}

Se trata de un estudio descriptivo, transversal y comparativo.

\section{Participantes}

En la investigación participaron alumnos españoles y mexicanos de ESO. Los españoles eran de Almería, Granada y Murcia, mientras que los mexicanos eran de Monterrey. Para asegurar una muestra representativa de ambas poblaciones (error $= \pm 3 \%$, IC $=95,5 \%$ ), se empleó un muestreo polietápico estratificado por afijación proporcional y por conglomerados, con las siguientes consideraciones generales: tipo de centro (públicoprivado), curso $\left(1^{\circ}, 3^{\circ} / 4^{\circ}\right.$ de ESO) y sexo. Además, en España también se consideró el número de habitantes de las poblaciones (+50 000, 50 000-20 001, 20 000-10 001, 10 000-5 001, 5 000-2 001 y menos de 2001 habitantes). En México, por su parte, se consideró el horario escolar (turno matutino o vespertino).
En España se partió del total de los 137453 estudiantes matriculados para el curso 2005-2006 (Delegación Provincial de Educación de Almería, Granada y Murcia). En México se partió de los 57069 estudiantes matriculados para el curso 2006-2007 en Monterrey (Dirección de Educación Física y Deporte, Estado de Nuevo León-México).

Participaron 3249 alumnos españoles (1 701 varones, 52,4\%; y 1548 mujeres, $47,6 \%)$, con un rango de edad de entre 12 y 16 años (media $[\mathrm{M}]=14,12$; desviación típica $[\mathrm{DT}]=1,30)$ y 1083 alumnos mexicanos (535 varones, $49,4 \%$ y 548 mujeres, $50,6 \%)$, con un rango de edad de entre 12 y 16 años $(M=13,70 ; \mathrm{DT}=0,95)$.

\section{Instrumento}

Para obtener los datos se empleó la técnica de la encuesta, que permite extrapolar los resultados al total de adolescentes escolarizados de ambas naciones. Se utilizó un cuestionario ad hoc al que se le aplicaron las correspondientes pruebas de validez de contenido y constructo (juicio de expertos en la materia y psicometría) (33). Se constató su fiabilidad mediante cuatro estudios piloto con el fin de comprobar si los entrevistados comprendían las preguntas, la terminología y el vocabulario.

Partiendo del cuestionario, se seleccionaron para este estudio solamente las variables que evalúan el sexo y la edad; el consumo habitual de tabaco (frecuencia y unidades) (34) y de alcohol (frecuencia y unidades) (35); la implicación en actividades físico-deportivas de tiempo libre (activo, abandono, nunca haber practicado); el patrón de actividad físico-deportiva (8); la modalidad deportiva (individual o colectiva); la finalidad (lúdica o competitiva) y la motivación por la actividad. Además, se tuvo en cuenta el consumo de tabaco y alcohol en el entorno familiar (padre, madre, hermano/s, hermana/s).

La frecuencia de consumo de tabaco se estructuró en: no consumo, consumo esporádico u ocasional y consumo habitual, empleando un método previamente utilizado internacionalmente (35). Los fumadores debían indicar cuántos cigarrillos fumaban al día de promedio, agrupándose en tres categorías: de 1 a 5, de 6 a 10, y más de 11. El consumo de tabaco de padres, madres, hermano/s y hermana/s ( $\alpha=0,84$, España; $\alpha=0,86$, México) se evaluó mediante preguntas cerradas (de respuesta Sí/No). 
Con respecto a la frecuencia en el consumo de alcohol se fijaron tres categorías: no consumo, consumo esporádico y consumo diario (35). Se planteó a los bebedores que pensaran en la última ingestión de alcohol e indicaran el número de bebidas y tipo, calculándose posteriormente las unidades de alcohol consumidas ( 1 unidad de bebida estándar equivale a 8 gramos de alcohol). Se establecieron tres grupos: de 1 a 3, de 4 a 6, y 7 o más unidades de alcohol (36). Las conductas de consumo de alcohol del padre, madre, hermano/s o hermana/s se midieron con preguntas cerradas y presentaron buena fiabilidad ( $\alpha=0,88$, España; $\alpha=0,87$, México).

Los patrones de práctica de actividad físico-deportiva de los individuos activos se calcularon mediante cinco preguntas referidas en el índice de cantidad de actividad físico-deportiva citado en la bibliografía (8). Dichas preguntas se refieren a la frecuencia, la duración y la intensidad de la actividad física en el tiempo libre y a la participación en deportes organizados y en competiciones deportivas. Los resultados más bajos son característicos de las personas menos activas, mientras que las puntuaciones más altas son indicativas de los individuos más activos. El alfa de Cronbach demuestra una aceptable fiabilidad del conjunto de estas variables $(\alpha=0,82$, España; $\alpha=0,81$, México) y se asemeja a la obtenida en los trabajos citados. Para representar mejor los patrones de actividad de los participantes, según los trabajos mencionados, estos se clasificaron en actividad vigorosa, moderada, ligera e insuficiente.

La motivación se valoró mediante una escala de motivos para la práctica deactividad físico-deportiva, y presentó una consistencia interna adecuada $(\alpha=0,83$, España; $\alpha=0,82$, México). El modelo puesto a prueba, en análisis factorial confirmatorio, supone la existencia de dos variables latentes independientes (motivaciones extrínseca e intrínseca), que subyacen a las 20 variables o ítems. Los re-sultados muestran adecuados índices de ajuste en ambas muestras (España: $\chi^{2} / \mathrm{gl}=1,83$; GFI [índice de bondad de ajuste] $=0,94$; IFI [índice de ajuste incremental] $=0,93$; CFI [índice de ajuste comparativo] $=0,94$; TLI [índice de Tucker Lewis] =0,93; SRMR [raíz cuadrática media de residuales] = 0,038; RMSEA [error cuadrático medio de aproximación] $=0,061$. México: $\chi^{2} / \mathrm{gl}=2,87 ; \mathrm{GFI}=0,92 ; \mathrm{IFI}=0,91$; CFI =
0,93; TLI $=0,91 ;$ SRMR $=0,040 ;$ RMSEA $=$ $0,064)$.

\section{Procedimiento}

Se pidió permiso a los centros educativos, se explicaron los objetivos y el modo en el que se realizaría la investigación y se aportó el modelo del instrumento. Posteriormente, tras establecer un consenso entre escuelas y ofrecer formación a los evaluadores, se administró el cuestionario con aplicación masiva y se completó de manera anónima. En la investigación participaron solamente los alumnos que contaban con el consentimiento informado de los progenitores y tutores, y fueron informados del objetivo del estudio. Su participación fue voluntaria, y los datos obtenidos, confidenciales; sólo se utilizarían en el análisis de resultados y conclusiones. Se advirtió a los alumnos que no había respuestas correctas o incorrectas, y se les solicitó que contestaran con la máxima sinceridad y honestidad. En España, el estudio se realizó entre febrero y mayo de 2006; en México, entre abril y mayo de 2007. Este trabajo posee un informe favorable de la Comisión de Bioética de la Universidad de Murcia (España) y de la comisión de investigación de la Facultad de Organización Deportiva de la Universidad Autónoma de Nuevo León, Monterrey (México).

\section{Análisis estadísticos}

Se utilizó el paquete estadístico SPSS 17.0 (SPSS Inc., Chicago, IL, USA) para realizar el análisis descriptivo y ajustar el efecto de posibles elementos de confusión; se empleó regresión logística multinomial para averiguar la capacidad predictiva de cada variable incluida. La relación entre consumo de tabaco y alcohol, con motivación extrínseca e intrínseca, se analizó por medio de ANOVA.

\section{RESULTADOS}

\section{Descripción de los datos}

En el cuadro 1 se observa que en ambos países existe un porcentaje muy

CUADRO 1. Prevalencia de las características de los participantes, 2006-2007

\begin{tabular}{|c|c|c|c|c|}
\hline \multirow[b]{2}{*}{ Característica } & \multicolumn{2}{|c|}{$\begin{array}{c}\text { Total } \\
\text { Alumnos de ESO }{ }^{a} \text { españoles }\end{array}$} & \multicolumn{2}{|c|}{$\begin{array}{c}\text { Total } \\
\text { Alumnos de ESO mexicanos }\end{array}$} \\
\hline & No. & $\%$ & No. & $\%$ \\
\hline \multicolumn{5}{|l|}{ Sexo } \\
\hline Varón & 1701 & 52,4 & 535 & 49,4 \\
\hline Mujer & 1548 & 47,6 & 548 & 50,6 \\
\hline Total & 3249 & 100,0 & 1083 & 100,0 \\
\hline \multicolumn{5}{|l|}{ Edad (años) } \\
\hline 12 & 445 & 13,7 & 114 & 10,5 \\
\hline 13 & 670 & 20,6 & 357 & 33,0 \\
\hline 14 & 765 & 23,5 & 352 & 32,5 \\
\hline 15 & 787 & 24,2 & 234 & 21,6 \\
\hline 16 & 582 & 17,9 & 26 & 2,4 \\
\hline Total & 3249 & 100,0 & 1083 & 100,0 \\
\hline \multicolumn{5}{|c|}{$\begin{array}{l}\text { Comportamiento ante la práctica físico- } \\
\text { deportiva }\end{array}$} \\
\hline Nunca & 348 & 10,7 & 103 & 9,5 \\
\hline Abandono & 838 & 25,8 & 260 & 24,0 \\
\hline Activo & 2063 & 63,5 & 720 & 66,5 \\
\hline Total & 3249 & 100,0 & 1083 & 100,0 \\
\hline \multicolumn{5}{|c|}{ Patrón de actividad físico-deportiva } \\
\hline Sedentario & 1186 & 36,5 & 363 & 33,5 \\
\hline Insuficiente & 218 & 6,7 & 108 & 10,0 \\
\hline Ligera & 416 & 12,8 & 160 & 14,8 \\
\hline Moderada & 820 & 25,2 & 266 & 24,6 \\
\hline Vigorosa & 609 & 18,7 & 186 & 17,2 \\
\hline Total & 3249 & 100,0 & 1083 & 100,0 \\
\hline \multicolumn{5}{|l|}{ Modalidad deportiva } \\
\hline Deportes colectivos & 1382 & 67,0 & 479 & 66,5 \\
\hline Deportes individuales & 681 & 33,0 & 241 & 33,5 \\
\hline Total & 2063 & 100,0 & 720 & $\begin{array}{l}100,0 \\
\text { (continúa) }\end{array}$ \\
\hline
\end{tabular}




\begin{tabular}{|c|c|c|c|c|}
\hline \multirow[b]{2}{*}{ Característica } & \multicolumn{2}{|c|}{$\begin{array}{l}\text { Total } \\
\text { Alumnos de ESO }{ }^{\mathrm{a}} \text { españoles }\end{array}$} & \multicolumn{2}{|c|}{$\begin{array}{c}\text { Total } \\
\text { Alumnos de ESO mexicanos }\end{array}$} \\
\hline & No. & $\%$ & No. & $\%$ \\
\hline \multicolumn{5}{|l|}{ Finalidad de la práctica } \\
\hline Competición & 879 & 42,6 & 304 & 42,2 \\
\hline Sin carácter competitivo & 1184 & 57,4 & 416 & 57,8 \\
\hline Total & 2063 & 100,0 & 720 & 100,0 \\
\hline \multicolumn{5}{|l|}{ Consumo de alcohol } \\
\hline Nunca & 2125 & 65,4 & 967 & 89,3 \\
\hline Ocasional & 278 & 8,6 & 53 & 4,9 \\
\hline Habitual & 846 & 26,0 & 63 & 5,8 \\
\hline Total & 3249 & 100,0 & 1083 & 100,0 \\
\hline \multicolumn{5}{|l|}{ Unidades de consumo } \\
\hline $1-3$ & 392 & 44,2 & 34 & 57,6 \\
\hline $4-6$ & 210 & 23,7 & 15 & 16,9 \\
\hline$\geq 7$ & 285 & 32,1 & 34 & 25,4 \\
\hline No contesta & 237 & - & 33 & - \\
\hline Total & 1124 & 100,0 & 116 & 100,0 \\
\hline \multicolumn{5}{|l|}{ Consume alcohol el padre } \\
\hline Sí & 1978 & 61,9 & 722 & 67,8 \\
\hline No & 1220 & 38,1 & 343 & 32,2 \\
\hline Total & 3198 & 100,0 & 1065 & 100,0 \\
\hline \multicolumn{5}{|l|}{ Consume alcohol la madre } \\
\hline Sí & 1047 & 37,0 & 271 & 26,5 \\
\hline No & 1782 & 63,0 & 752 & 73,5 \\
\hline Total & 2829 & 100,0 & 1023 & 100,0 \\
\hline \multicolumn{5}{|l|}{ Consumen alcohol los hermanos } \\
\hline Sí & 864 & 32,5 & 190 & 20,5 \\
\hline No & 1798 & 67,5 & 737 & 79,5 \\
\hline Total & 2662 & 100,0 & 927 & 100,0 \\
\hline \multicolumn{5}{|l|}{ Consumen alcohol las hermanas } \\
\hline Sí & 473 & 22,1 & 81 & 9,4 \\
\hline No & 1671 & 77,9 & 783 & 90,6 \\
\hline Total & 2144 & 100,0 & 864 & 100,0 \\
\hline \multicolumn{5}{|l|}{ Consumo de tabaco } \\
\hline Nunca & 2758 & 84,9 & 1017 & 93,9 \\
\hline Ocasional & 260 & 8,0 & 28 & 2,6 \\
\hline Habitual & 231 & 7,1 & 38 & 3,5 \\
\hline Total & 3249 & 100,0 & 1083 & 100,0 \\
\hline \multicolumn{5}{|l|}{ Cigarrillos semanales } \\
\hline $1-5$ & 222 & 54,1 & 38 & 61,3 \\
\hline $6-10$ & 114 & 27,8 & 14 & 22,5 \\
\hline$\geq 11$ & 74 & 18,0 & 10 & 16,2 \\
\hline No contesta & 81 & - & 4 & - \\
\hline Total & 491 & 100,0 & 66 & 100,0 \\
\hline \multicolumn{5}{|l|}{ Consume tabaco el padre } \\
\hline Sí & 2057 & 64,3 & 510 & 48,3 \\
\hline No & 1143 & 35,7 & 546 & 51,7 \\
\hline Total & 3200 & 100,0 & 1056 & 100,0 \\
\hline \multicolumn{5}{|l|}{ Consume tabaco la madre } \\
\hline Sí & 1215 & 41,5 & 237 & 23,0 \\
\hline No & 1712 & 58,5 & 793 & 77,0 \\
\hline Total & 2927 & 100,0 & 1030 & 100,0 \\
\hline \multicolumn{5}{|l|}{ Consumen tabaco los hermanos } \\
\hline Sí & 943 & 35,2 & 154 & 16,5 \\
\hline No & 1739 & 64,8 & 781 & 83,5 \\
\hline Total & 2682 & 100,0 & 935 & 100,0 \\
\hline \multicolumn{5}{|l|}{ Consumen tabaco las hermanas } \\
\hline Sí & 453 & 20,1 & 76 & 8,8 \\
\hline No & 1799 & 79,9 & 791 & 91,2 \\
\hline Total & 2252 & 100,0 & 867 & 100,0 \\
\hline
\end{tabular}

Fuente: Elaborado a partir de la base de datos construida mediante SPSS 17.0.

Nota: No se encontraron diferencias significativas entre estudiantes mexicanos y españoles en ninguna de las variables analizadas.

a ESO: Educación Secundaria Obligatoria. elevado de sujetos sedentarios. Las personas físicamente activas prefieren los deportes colectivos a los individuales, y predominan las actividades lúdicas ante las competitivas.

El porcentaje de personas activas es ligeramente mayor en México, aunque estas presentan un patrón de actividad físico-deportiva más bajo. En todos los casos, las diferencias no fueron estadísticamente significativas $(P>0,05)$.

El consumo de alcohol es mayor en España, y también es superior el número de unidades consumidas por persona $(P<0,001)$. La misma observación se encontró respecto del consumo de tabaco: fue mayor el número de fumadores españoles, y mayor el número de cigarrillos consumidos $(P<0,001)$.

En relación con el entorno familiar, tanto en España como en México los padres son la figura familiar que más alcohol y tabaco consumen, seguidos de las madres, los hermanos y las hermanas.

\section{Variables de predicción de la frecuencia de consumo}

En el cuadro 2 se observa que en España ser mujer es un factor de riesgo en el consumo frecuente de bebidas alcohólicas, que muestra mayor probabilidad que en los varones. En México ocurre a la inversa; la ingestión frecuente de bebidas alcohólicas es más frecuente en los varones.

Respecto del tabaco, en España ocurre algo semejante: se aprecia una mayor probabilidad de consumo entre las alumnas. En México, en cambio, no se encontraron diferencias notorias al respecto.

En ambos países, a medida que aumenta la edad se acrecienta la probabilidad de consumir alcohol y tabaco con más frecuencia. Este dato es más destacado en España donde, a mayor edad, mayor cantidad de unidades ingeridas.

La relación directa entre el consumo de alcohol o tabaco y los comportamientos ante la práctica físico-deportiva, sólo arroja diferencias significativas en España; tanto en el consumo habitual como en el esporádico, las personas físicamente inactivas fuman y beben más que las activas.

En España, respecto del patrón de actividad físico-deportiva, su intensidad y sus fines, se comprueba que al mismo tiempo que disminuye el grado de energía de la práctica, aumenta la probabilidad 


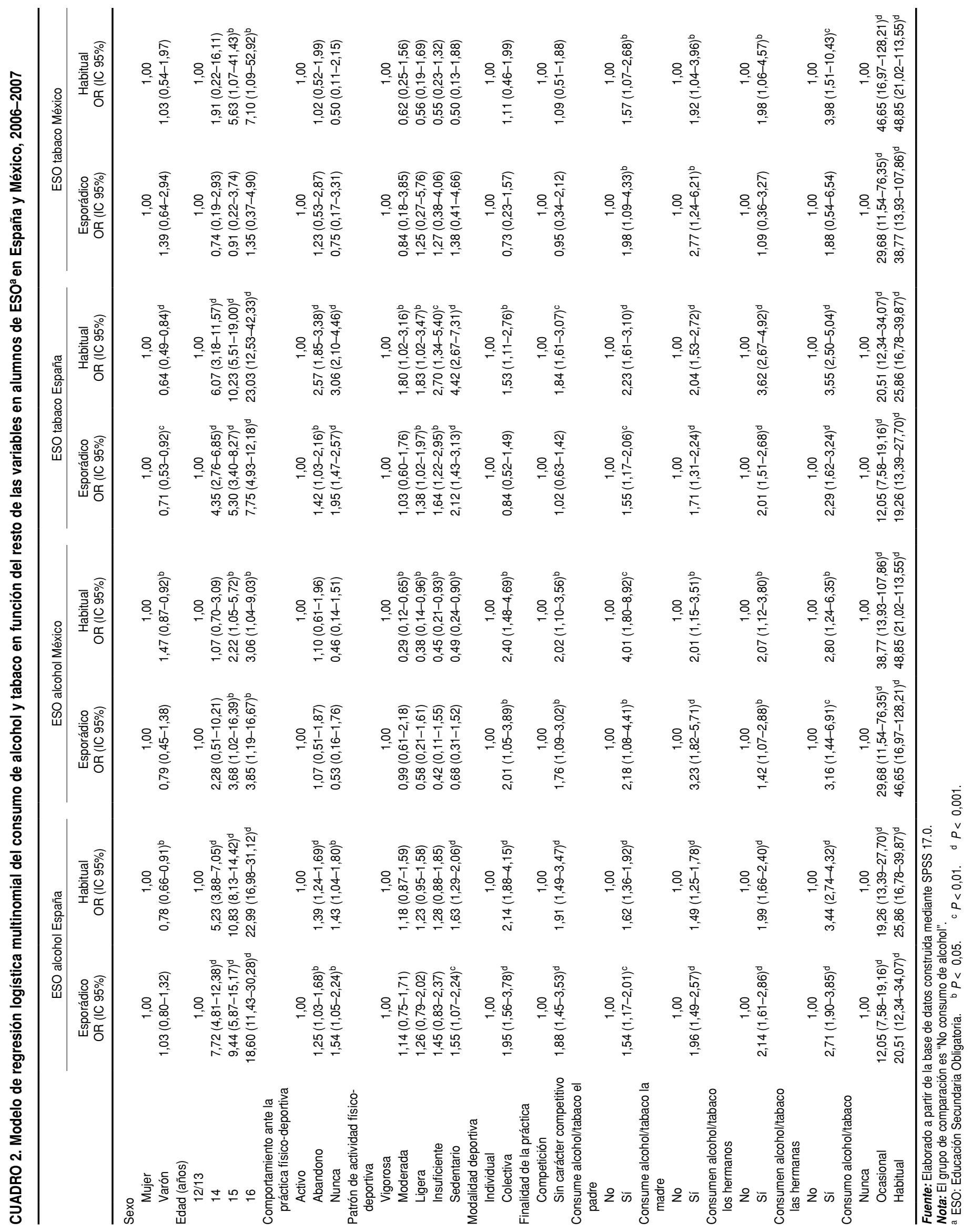


de ingerir alcohol; en México, no obstante, a mayor intensidad, mayor probabilidad de consumo. Los alumnos españoles muestran una relación inversamente proporcional entre la probabilidad de fumar y la intensidad del ejercicio deportivo. Entre los mexicanos no se observan diferencias importantes. En los dos países, el hecho de practicar deportes colectivos sin fines competitivos se manifiesta como un factor de riesgo para el consumo de alcohol.

El ANOVA no arroja diferencias comparativas estadísticamente evidentes entre el consumo de alcohol o tabaco y la motivación extrínseca por la práctica deportiva; aun así, la motivación intrínseca es mayor entre los no fumadores y los abstemios.

El contexto familiar constituye un factor de riesgo patente. En relación con el alcohol, los familiares con mayor influencia en España son las hermanas y, en México, los padres. Respecto del tabaco, los familiares que con más frecuencia influyen negativamente son los hermanos, en España, y las hermanas, en México.

En cuanto al consumo simultáneo de ambas sustancias, el porcentaje de alumnos mexicanos duplica al de españoles. Se demostró que, en ambos países, los fumadores tienen más tendencia a beber de forma habitual.

\section{Intensidad de consumo y variables de predicción}

En el cuadro 3 se observa que los estudiantes tienen mayor probabilidad de consumir alcohol y tabaco que las estudiantes. En España, a diferencia de en México, al aumentar la edad también lo hace la posibilidad de consumir sustancias nocivas en mayores cantidades.

Ni los comportamientos hacia la práctica físico-deportiva; ni el patrón, la modalidad, o finalidad de la actividad; ni la motivación extrínseca o intrínseca parecen guardar ninguna relación con la intensidad de consumo, ya que no existen diferencias numéricas destacables.

En las familias españolas, el padre que consume alcohol habitualmente es quien más influencia ejerce en los estudiantes que consumen 7 o más unidades de alcohol; las hermanas no tienen influencia alguna. En México, la mayor influencia se debe a los hermanos y las hermanas. No existen diferencias comparativas respecto del tabaco.
Los adolescentes fumadores, tanto españoles como mexicanos, tienen mayor probabilidad de consumir más alcohol que los no fumadores. Se observa una relación directa con el número de cigarrillos consumidos solamente en los estudiantes españoles; los adolescentes que consumen alcohol habitualmente tienen más posibilidades de consumir entre $6 \mathrm{y}$ 10 cigarrillos.

\section{DISCUSIÓN}

Al igual que otros estudios realizados (37-40), esta investigación muestra la existencia de un gran número de adolescentes españoles consumidores de alcohol y tabaco, lo que evidencia una gran prevalencia de consumo de estas sustancias adictivas. Por el contrario, y también en concordancia con otros estudios similares $(4,9,41)$, entre los adolescentes mexicanos las cifras de consumo no resultan tan alarmantes en términos comparativos. Por tanto, contamos con evidencias que indican conductas más saludables en los adolescentes mexicanos que en los españoles.

En España, es mayor la probabilidad de que las adolescentes beban y fumen con mayor frecuencia, y la de que los adolescentes lo hagan en mayores cantidades, datos que coinciden con los de estudios previos (42, 43). En México encontramos que los varones consumen alcohol más a menudo, un dato también comunicado en investigaciones anteriores llevadas a cabo en América Latina $(3,9,30)$. Estas diferencias de consumo en función del sexo entre ambos países podrían deberse a la mayor integración social de la mujer en los países europeos occidentales, en comparación con los países en vías de desarrollo.

Diferentes estudios han demostrado que la edad es un factor de riesgo para el consumo de sustancias adictivas; la probabilidad de consumirlas con mayor frecuencia y en mayores cantidades aumenta con el tiempo $(4,9,16,44,45)$. Esta investigación ha corroborado estos datos. No obstante, y a modo comparativo, se observan grandes diferencias, y entre los españoles las cifras de probabilidad de consumo fueron mucho más altas. Esto podría deberse al fenómeno social denominado "botellón" (reunión de jóvenes para consumir alcohol en la vía pública), que hace del consumo de alcohol y tabaco un hábito cada vez más precoz (46, 47). La aceptación social de estas sus- tancias en Europa y las facilidades para adquirirlas hace que los adolescentes españoles estén más desprotegidos.

Los adolescentes españoles activos tienen menos probabilidades de consumir alcohol y tabaco que los sedentarios; estos resultados concuerdan con los de otros estudios españoles y europeos. Ser activo ejerce en los adolescentes españoles un efecto protector y preventivo frente a conductas poco saludables, mientras que ser sedentario es un factor de riesgo para la frecuencia de consumo de sustancias nocivas (13-16). En México, por su parte, ser activo no se relaciona con una menor predisposición al consumo.

A pesar de que algunas teorías afirman que la práctica físico-deportiva podría potenciar la aparición de hábitos insalubres (17), la gran mayoría da por cierta la idea de que, incorporada a temprana edad, puede tener efectos a largo plazo y perdurar hasta la madurez, influyendo de manera positiva en la salud general de las poblaciones $(6,8,12-16)$.

En el análisis solamente de los sujetos activos, encontramos que practicar deportes colectivos deriva en una mayor prevalencia de consumo de alcohol, en concordancia con otros estudios americanos y europeos (18-20). Los adolescentes que practican deportes individuales lo hacen en menor medida. La explicación podría estar relacionada con la teoría de la autodeterminación (2426). Las necesidades personales de autonomía, competencia y relación se ven condicionadas bien por factores socioculturales o extrínsecos (hacer amigos, sentirse miembro de un grupo o lograr aceptación social), o bien por factores personales o intrínsecos (sentir placer y satisfacción por practicar). Los jóvenes que optan por modalidades colectivas declaran en mayor medida sentirse atraídos por motivos extrínsecos, lo que coincide con los motivos reflejados en otras investigaciones (48-50). En España, los participantes en deportes colectivos tienen más probabilidad de convertirse en fumadores. Por otro lado, el carácter competitivo de la actividad físico-deportiva lleva a una menor prevalencia de consumo de alcohol en ambos países y a una menor prevalencia de consumo de tabaco en España. Otros estudios similares no habían encontrado relación entre la finalidad de la práctica y el consumo de alcohol y tabaco (51). Según este 


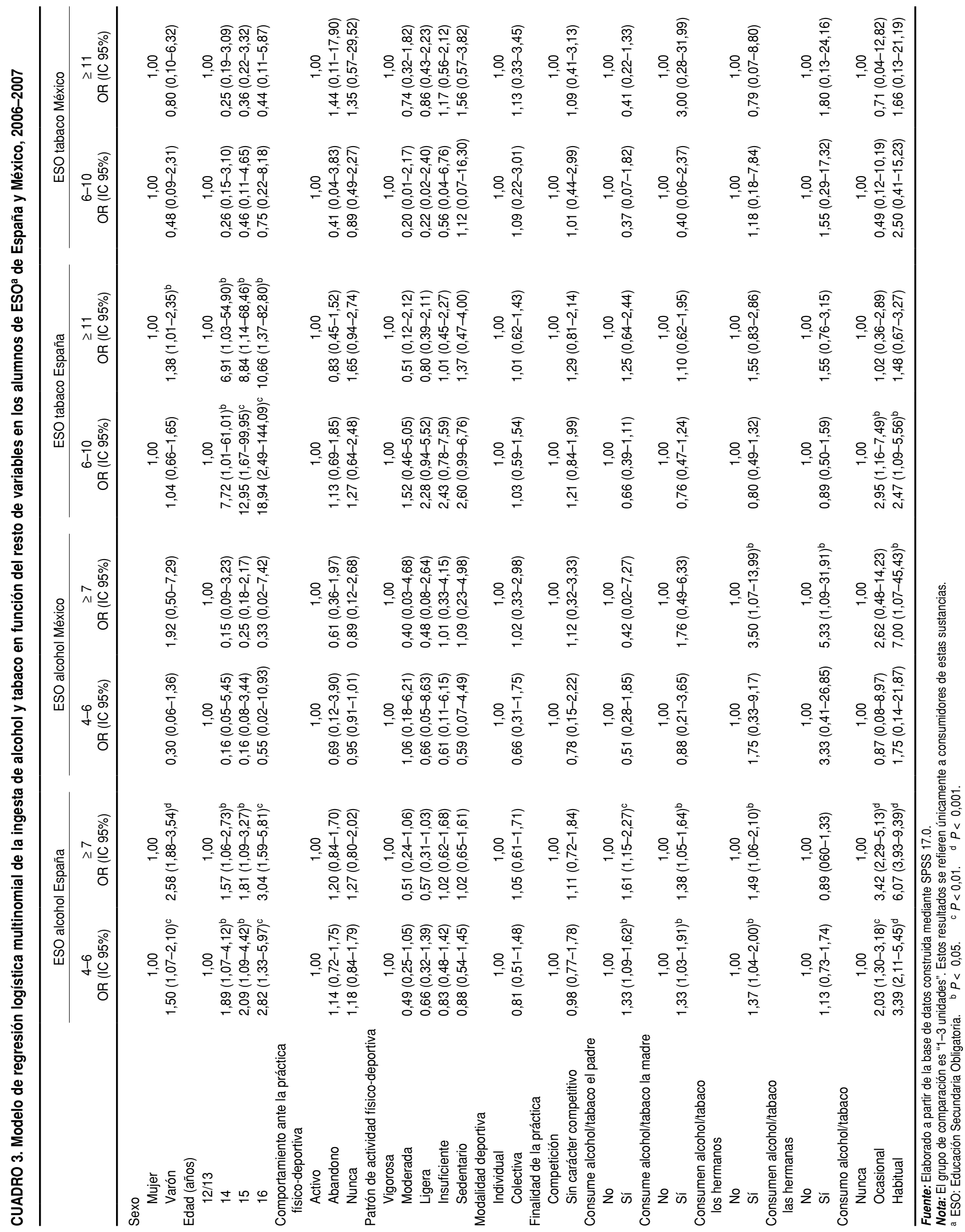


estudio, practicar de manera reglada y con fines competitivos podría considerarse una solución saludable contra el consumo de sustancias nocivas.

En ambas muestras analizadas, tal como consignaron otros estudios (4, 27-28), las familias juegan un papel muy importante en el consumo. Los padres tienen mayor influencia durante la niñez y adolescencia, pero esta disminuye al acercarse a la edad adulta. La presencia de hermanos con conductas adictivas hacia el consumo de alcohol y tabaco es un alarmante factor de riesgo $(4,52)$. Considerados como un grupo "especial" de compañeros, ejercen un modelado directo sobre la conducta, las actitudes y las orientaciones de los más jóvenes.

Por último, cabe destacar el alto índice existente de consumo simultáneo de las dos sustancias, con una relación directamente proporcional entre la frecuencia de consumo de tabaco y alcohol. Entre $80 \%$ y $95 \%$ de las personas alcohólicas fuman, lo que demuestra el grado de dependencia de los fumadores hacia el alcohol $(31,32)$. En México, el consumo conjunto es casi el doble del observado en España, lo que pone en evidencia la baja percepción de riesgo que podrían tener los adolescentes mexicanos.
Este estudio presenta diversas limitaciones, que podrían subsanarse en futuras investigaciones con un punto de vista longitudinal y mediante la utilización de autoinformes. Quizá, y debido a su carácter transversal, queda limitada la posibilidad de establecer una direccionalidad en las asociaciones observadas. No se han tenido en cuenta otras variables que podrían haber afectado a los resultados como, por ejemplo, la influencia de amigos. Otra de las limitaciones podría ser la falta de veracidad de las respuestas de los encuestados.

En conclusión, se han encontrado conductas más saludables en adolescentes mexicanos. Existen diferencias de consumo en función del sexo; las adolescentes españolas beben y fuman con mayor frecuencia, pero en menores cantidades que los adolescentes. En México, los varones consumen alcohol con mayor frecuencia que las mujeres, sin diferencias en la cantidad. La edad es un factor de riesgo en ambos países. En España, la práctica físico-deportiva se asocia con una menor prevalencia de consumo; ser físicamente activo actúa como un factor de protección. Este fenómeno no es significativo en los estudiantes mexicanos. Por su parte, realizar modalidades deportivas colectivas conlleva un mayor consumo de alcohol. En los dos países, la finalidad competitiva del deporte implica una menor prevalencia del consumo de alcohol; en España esto también sucede con el tabaco. Los hermanos y los padres, tanto en España como en México, son los miembros de la familia más influyentes respecto de los hábitos insalubres. Existe una relación clara y directamente proporcional entre el consumo conjunto de alcohol y tabaco, lo que genera un grado de dependencia recíproca y ejerce efectos devastadores en la salud.

Los resultados de este estudio son de gran utilidad a la hora de comprobar el efecto que la práctica físico-deportiva y el entorno socioafectivo tienen en la adquisición y regulación de conductas saludables o nocivas.

Financiación. Este estudio es resultado del subproyecto "Factores determinantes para la práctica físico-deportiva y asociaciones probabilísticas entre variables físico-deportivas y de salud. Tipologías de estilos de vida de la población adulta y detección de poblaciones con riesgo para la salud" (DEP200500231-C03-02/ACTI), subvencionado por el Ministerio de Educación, Cultura y Deporte, España (Plan Nacional I+D+I: 2004-2007).

\section{REFERENCIAS}

1. Organización Mundial de la Salud. Estrategias para reducir el uso nocivo del alcohol. Ginebra: OMS; 2008.

2. Delegación del Gobierno para el Plan Nacional sobre Drogas. Observatorio Español sobre Drogas. Situación y tendencias de los problemas de drogas en España. Madrid: DGPNSD; 2009.

3. Consejo Nacional sobre Adicciones. Observatorio mexicano en tabaco, alcohol y otras drogas. México. D.F.: CNA; 2003.

4. Urquieta JE, Hernández-Ávila M, Hernández B. El consumo de tabaco y alcohol en jóvenes de zonas urbanas marginadas de México. Un análisis de decisiones relacionadas. Salud Publica Mex. 2006;48(1):30-40.

5. Paavola M, Vartiainen E, Haukkala A. Smoking, alcohol use, and physical activity: A 13-year longitudinal study ranging from adolescence into adulthood. J Adolescent Health. 2004;35(3):238-44.
6. Ruiz-Juan F, Ruiz-Risueño Abad J, De la Cruz-Sánchez E, García-Montes M. Physical activity and alcohol consumption in adults. Retos. 2009;16:46-50.

7. Irwin CE. Eating and physical activity during adolescence: Does it make a difference in adult health status? J Adolesc Health. 2004; 34(6):459-60.

8. Telama R, Yang X, Viikari J, Välimäki I, Wanne O, Raitakari O. Physical activity from childhood to adulthood: A 21-year tracking study. Am J Prev Med. 2005;28(3):267-73.

9. Herrera-Vázquez M, Wagner FA, VelascoMondragón E, Borges G, Lazcano-Ponce E. Inicio en el consumo de alcohol y tabaco y transición a otras drogas en estudiantes de Morelos, México. Salud Publica Mex. 2004;46: $132-40$.

10. Patrones de consumo de tabaco en estudiantes de 13 a 15 años. Rev Panam Salud Publica. 2006;19(3):198-9.
11. Rooney JF. Sports and clean living: A useful myth? Drug Alcohol Depend. 1984;13(1): 75-87.

12. Arruza JA, Arribas S, Gil DL, Irazusta S, Romero S, Cecchini JA. The impact of duration in sport and physical activity on the psychological well-being. Rev Int Med Cienc Act Fís Deporte. 2008;8(30): $171-83$.

13. Ruiz-Risueño J. Sporting habits and lifestyles of the schoolchildren of Secondary Obligatory Education in the municipalities of the province of Almería. Retos. 2010; 17:49-53.

14. Hermoso, Y, García V, Chinchilla J. Study of the participation of schoolchildren in leisure time. Retos. 2010;18:9-13.

15. Ferez S, Pappous A, Ruffié S. Activité physique et sportive des PVVIH : de l'intérêt de la pratique à l'accès à la pratique (revue de littérature). Retos. 2010;18:77-83. 
16. Ruiz F, De la Cruz E, Ruiz-Risueño J, García M. Youth smoking patterns and leisure-time physical activity. Retos. 2008;14:75-9.

17. Rainey CJ, McKeown RE, Sargent RG, Valois RF. Patterns of tobacco and alcohol use among sedentary, exercising, nonathletic, and athletic youth. J Sch Health. 1996;66(1): 27-32.

18. Moore MJ, Werch CEC. Sport and physical activity participation and substance use among adolescents. J Adolesc Health. 2005;36(6): 486-93.

19. Ford JA. Substance use among college athletes: A comparison based on sport/team affiliation. J Am Coll Health. 2007;55(6):367-73.

20. Lorente FO, Souville M, Griffet J, Grélot L. Participation in sports and alcohol consumption among French adolescents. Addict Behav. 2004;29(5):941-6.

21. Alves R, Kososbudzky LA. Caracterizacao dos adolescentes internados por alcool e otras drogas na Cidade de Cutritiba. Interacao em Psicologia. 2002;6(1):65-79.

22. Carvalho A, Lemos E, Raimundo F, Costa M, Cardoso F, Sousa MC, et al. Consumo de substancias psicoactivas em adolescentes de uma Escola Secundaria com $3^{\circ}$ Ciclo: que realidade? Instituto Nacional de Investigación Científica, 2003.

23. Fraile DCG, Riquelme PN, Pimenta CA. Consumo de Drogas lícitas e ilícitas en escolares y factores de protección y de riesgo. Revista Latinoamericana de Enfermagem, Ribeirao Preto. 2004;12:345-51.

24. Deci E, Ryan R. Intrinsic motivation and selfdetermination in human behavior. New York: Plenum Publishing Co.; 1985.

25. Deci E L, Ryan R M. The "what" and "why" of goal pursuits: Human needs and the selfdetermination of behavior. Psychol Inq. 2000; 11:227-68.

26. López-Walle J, Balaguer I, Castillo I, Tristán J. Clima motivacional percibido, motivación autodeterminada y autoestima en jóvenes deportistas mexicanos. Rev Psicol Deporte. 2011;20(1):209-22

27. Pons, J. El modelado familiar y el papel educativo de los padres en la etiología del consumo de alcohol en los adolescentes. Rev Esp Salud Publica. 1998;72:251-66.

28. Espada JP, Pereira, JR, García-Fernández J. Influencia de los modelos sociales en el consumo de alcohol de los adolescentes, Psicothema. 2008;20(4):531-37.

29. Latimer W, Floyd LJ, Kariis T, Novotna G, Exnerova $\mathrm{P}, \mathrm{O}^{\prime}$ Brien $\mathrm{M}$. Peer and sibling sub- stance use: predictors of substance use among adolescents in Mexico. Rev Panam Salud Publica. 2004;15(4):225-32.

30. Verra F, Zabert G, Ferrante D, Morello P, Virgolini M. Consumo de tabaco en estudiantes de educación secundaria de Argentina. Rev Panam Salud Publica. 2009;25(3):227-33.

31. National Institute of Health. (1998). Alcohol Alert. National Institute on Alcohol Abuse and Alcoholism, 39. Disponible en http:// pubs.niaaa.nih.gov/publications/aa39.htm Acceso el 14 de marzo de 2009.

32. Daeppen JB, Smith TL, Danko GP, Gordon L, Landi NA, Nurnberger JI Jr, et al. Clinical correlates of cigarette smoking and nicotine dependence in alcohol-dependent men and women. The Collaborative Study Group on the Genetics of Alcoholism. Alcohol. 2000;35: 171-5.

33. Piéron M, Ruiz-Juan, F. Actividad físicodeportiva y salud. Análisis de los determinantes de la práctica en el alumnado de Enseñanza Secundaria. Madrid: Consejo Superior de Deportes; 2009.

34. Pérez-Milena A, Leal-Helmling FJ, JiménezPulido I, Mesa-Gallardo I, Martínez-Fernández ML, Pérez-Milen R. Evolution of the consumption of toxic substances among the adolescents of an urban area (1997-2004). Aten Primaria. 2007;39(6):299-304.

35. Lorente FO, Souville M, Griffet I, Grélot L. Participation in sports and alcohol consumption among French adolescents. Addict Behav. 2004;29(5):941-6.

36. Sommers MS. Measurement of alcohol consumption: issues and challenges. Annu Rev Nurs Res. 2005;23:27-64.

37. Ariza C, Nebot M. La prevención del tabaquismo en los jóvenes. Realidad y retos para el futuro. Adicciones. 2004;16(2).

38. España, Ministerio de Sanidad y Consumo. Informe de la Encuesta Estatal Sobre Uso de Drogas en Estudiantes de Enseñanzas Secundarias (ESTUDES) 2006-2007. Madrid: Ministerio de Sanidad y Consumo; 2007.

39. Espada JP, Méndez X, Griffin K, Botvin G. Adolescencia: Consumo de alcohol y otras drogas. Papeles del Psicólogo. 2003;84:9-17.

40. Sánchez L. Consumo de alcohol en la población juvenil. Adicciones. 2002;14(1).

41. Lopez-Garcia KS, Esparza-Almanza SE, Guzmán-Facundo FR, Alonso-Castillo MM, Rodríguez-Aguilar L. Expectativas del consumo de alcohol y tabaco en adolescentes escolares. Revista Científica Electrónica de Psicología. 2009;7:112-26.
42. Mendoza-Berjano R, Batista-Foguet, JM, Sánchez-García M, Carrasco-González A. M. The consumption of tobacco, alcohol and other drugs by adolescent Spanish students. Gac Sanit. 1998;12(6):263-71.

43. Hedman L, Bjerg-Bäcklund A, Perzanowski M, Sundberg S, Rönmark E. Factors related to tobacco use among teenagers. Respir Med. 2007;101(3):496-502.

44. Rodríguez J, De Abajo S, Márquez S. Relación entre actividad física y consumo de alcohol, tabaco y otras sustancias perjudiciales para la salud en alumnos de ESO del municipio de Avilés. Motricidad. 2004;12:46-69.

45. Gómez J.A, Fernández N, Romero E, Luengo A. El botellón y el consumo de alcohol y otras drogas en la juventud. Psicothema. 2008;20(2): 211-7.

46. Calafat A, Montserrat J, Becoña E, Castillo A, Fernández C, Franco M, Pereiro C, Ros M. El consumo de alcohol en la lógica del botellón. Adicciones. 2005;17(3):193-202.

47. Holmen TL, Barret-Connor E, Clausen J, Holmen J, Biermer L. Physical exercise, sports, and lung function in smoking versus non-smoking adolescents. Eur Respir Journal. 2002;19:8-15.

48. Kuntsche E, Knibbe R, Gmel G, Engels R. Why do young people drink? A review of drinking motives. Clin Psychol Rev. 2005;25(7):841-61.

49. Schuit AJ, van Loon AJM, Tijhuis M, Ocke MC. Clustering of lifestyle risk factors in a general adult population. Prev Med. 2002; 35(3):219-24

50. Gomez-Lopez M, Ruiz-Juan F, Garcia-Montes ME, Flores-Allende G, Barbero-Montesinos G. Reasons influencing physical inactivity-sport in post-compulsory secondary education. Retos. 2008;(14):80-5.

51. Ruiz-Juan F, De la Cruz-Sánchez E, GarcíaMontes M. Motivos para la práctica deportiva y su relación con el consumo de alcohol y tabaco en jóvenes españoles. Salud Publica Mexico. 2009;51(6):496-504.

52. Brook J, Whiteman M, Gordon A, Brook D. The Role of Older Brothers in Younger Brothers' Drug Use Viewed in the Context of Parent and Peer Influences. J Genet Psychol. 1989; 151(1):59-75.

Manuscrito recibido el 26 de noviembre de 2010. Aceptado para publicación, tras revisión, el 14 de junio de 2011 
ABSTRACT Objective. To identify the influence of alcohol or tobacco consumption along with independent variables (sex and age; time and leisure activities; motivations, patterns, modalities, and goals of physical and sports-related activity; and behaviors within the

Alcohol and tobacco consumption in Spanish and Mexican adolescents and its relation to physical and sports-related activity and to the family family environment) in physical and sports-related activity among pupils enrolled in compulsory secondary education in Spain and Mexico.

Methods. A questionnaire on habits related to physical and sports-related activity, consumption of alcoholic beverages or tobacco, and the influence of the family environment was answered by 3249 Spanish pupils and 1083 Mexican pupils between 12 and 16 years of age. A descriptive analysis and a multinomial logistic regression were carried out in order to ascertain the predictive capacity of the variables included in the model.

Results. In both countries, consumption increases with age. In comparative terms, there is a higher prevalence among Spanish pupils when consuming addictive substances. The Spanish pupils who are physically active are less likely to consume alcohol and tobacco; among their Mexican counterparts, there were no significant differences in this regard. The results show that in Spain, females consume alcoholic beverages and smoke more frequently than do males, who do so sporadically but in greater quantities. In Mexico, adolescent boys consume alcohol more regularly that do adolescent girls, although there are no differences in terms of quantity.

Conclusions. In Spain, being physically active and engaging in sports is clearly related to consuming less. Being physically active acts as a protective agent against unhealthy habits. Among Mexican adolescents, no significant relationship between these factors has been found. Both in Spain and in Mexico, siblings and parents are the family members who have the greatest influence vis-à-vis alcohol and tobacco consumption. Furthermore, the trend towards simultaneous consumption of both substances has been proved.

Key words Alcohol drinking; tobacco; adolescent; family; sports; Spain; Mexico. 